\title{
Implementation of Cognitive and Virtual Mouse Pointer Control
}

\author{
${ }^{1}$ Jayaprakash Nagaruru, ${ }^{2}$ V.Ravi Kumar \\ ${ }^{I} P G$ Scholar, ${ }^{2}$ Associate Professor \\ MRITS, Secunderabad
}

\begin{abstract}
Human Computer Interaction plays a key role in daily life like controlling Mouse operations, Keyboard operations controlling it in different ways like Physical, Cognitive, and Affective. In this Project We are Analyzing Human computer Interaction based on Virtual and Cognitive Technologies. Virtual Technology based on RGB Technique and Cognitive Mouse Control Based on RF Communication.
\end{abstract}

Keywords: HCI, Matlab, Image Processing RF Communication, Camera

\section{Introduction}

Existing Technologies in Human Computer Interaction are Physical, Cognitive and Affective. My Project Analysis is working on Mouse operations in Computers. In General we have seen a wired and wireless Mouse both Techniques are Single Purpose. We are working on two different ways 1. Virtual Mouse with RGB Techniques and 2.Cognitive Mouse

Controlling with Texas MSP430 microcontroller based on C1111 USB RF as Receiver and CC430 Transmitter.HCI design should consider many aspects of human behaviors and needs to be useful. The complexity of the degree of the involvement of a human in interaction with a machine is sometimes

Invisible compared to the simplicity of the interaction method itself. The existing interfaces differ in the degree of complexity both because of degree of functionality/usability and the financial and economical aspect of the machine in market.

The focus of this paper is mostly on the advances in physical aspect of interaction and to show how different methods of interaction can be combined (Multi-Modal Interaction) and how each method can be improved in performance (Intelligent Interaction) to provide a better and easier interface for the user. The existing physical technologies for HCI basically can be categorized by the relative human sense that the device is designed for. These devices are basically relying on three human senses: vision, audition, and touch. Input devices that rely on vision are the most used kind and are commonly either switch-based or pointing devices. The switch-based devices are any kind of interface that uses buttons and switches like a keyboard.

i)Virtual Mouse:

\section{Implementation Work}

Implementation of Virtual Mouse is worked out with the help of Matlab and Camera. The main Technique is RGB color based because Red, Green and Blue had sensing power, Where Red Color is used for Mouse Pointer Controlling, Blue Color is used for right click and left click and Green color is used Scrolling of Pages up and down.

\section{Following are the steps in our approach:}

- Capturing real time video using Web-Camera.

- Processing the individual image frame.

- Flipping of each image Frame.

- Conversion of each frame to a grey scale image.

- Color detection and extraction of the different colors (RGB) from flipped gray scale image

- Conversion of the detected image into a binary image.

- Finding the region of the image and calculating its centroid.

- Tracking the mouse pointer using the coordinates obtained from the centroid.

- Simulating the left click and the right click events of the mouse by assigning different color pointers. 


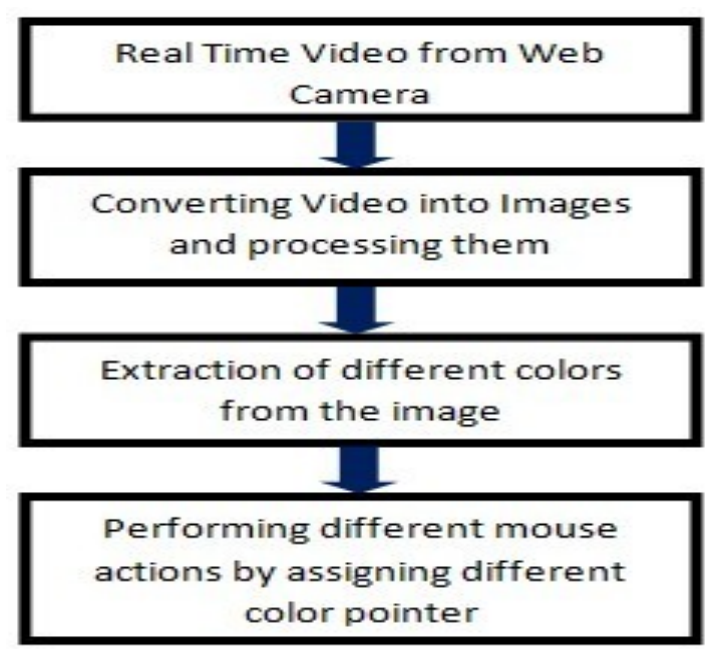

Figure 1: Block Diagram of the System.

\section{ii) Capturing the real time video:}

For the system to work we need a sensor to detect the hand movements of the user. The webcam of the computer is used as a sensor. The webcam captures the real time video at a fixed frame rate and resolution which is determined by the hardware of the camera. The frame rate and resolution can be changed in the system if required.

- Computer Webcam is used to capture the Real Time Video

- Video is divided into Image frames based on the FPS of the camera

- Processing of individual Frames.

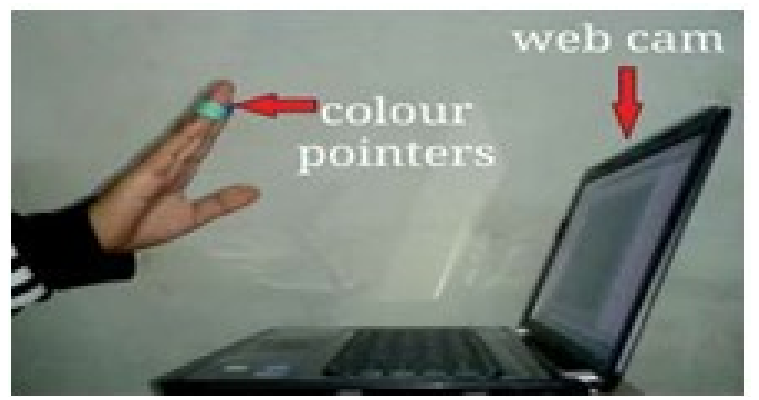

Figure 2: Capturing the Video

\section{Working}

As the related work shows, the choice of the algorithms chiefly depends on the environment in which the system is required to operate. A larger setup of the system in an open environment, such as a shopping mall or outdoors requires robust hardware that can cope with many users and difficult lighting conditions such as sunset. The choice of hardware to capture the users is a defining element of the reliability of the final system.

a) Image acquisition setup: It consists of a web camera with suitable interface for connecting it to PC.

b) Processor: It consists of personal computer or a dedicated image processing unit.

c) Image analysis: Certain tools are used to analyze the content in the image captured and derive conclusions e.g. MATLAB 12.0

d) Machine control: After processing, some conclusions have to be made in order to initiate control actions. In our case control actions are desktop control via mouse control. 


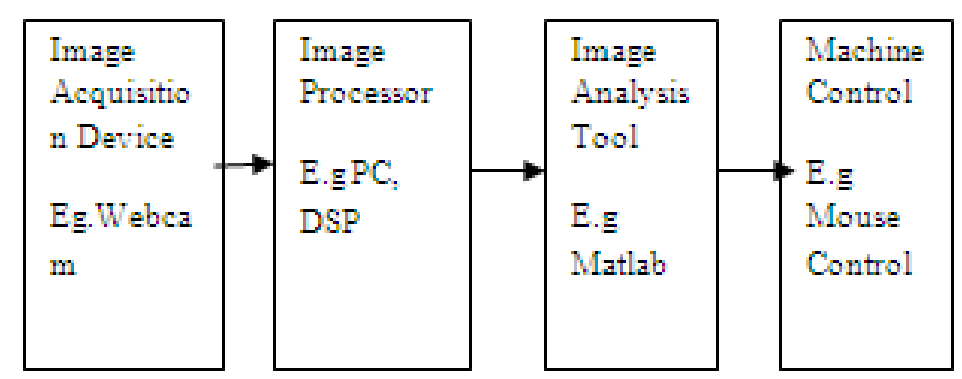

Figure 3: Block Diagram

\section{Implementation in image processing:}

As we all know, an image is defined as $2 \mathrm{D}$ function of $\mathrm{f}(\mathrm{x}, \mathrm{y})$ where $\mathrm{x} \& \mathrm{y}$ are spatial coordinates and amplitude at any pair of coordinates is called intensity of image at that point

1. Initialize the webcam and capture the image.

2. Then convert the captured colored image to gray scale image using image processing toolbox. The gray scale contains only brightness information about the image.

3. Later use image segmentation to locate objects and their boundaries in an image. In image segmentation, thresholding is their gray scale value. If pixel intensity is less than threshold, corresponding pixel is black in resulting image. If pixel value has intensity greater than threshold, the resultant pixel is white, which represents object

4. Thus binary Images contain only black and white pixels, which indicate that it keeps only the significant part of the mage and removes the noise.

5. Thus the required target is white and the surrounding background is black. This is how image processing is carried out only the significant part of the mage and removes the noise.

\section{Introduction to Proposed Model}

Here we are proposing a new model of virtual mouse with the help of Texas Instrument development board and we are trying to implement mouse control instead of color detection, we are trying to implement with the help of watch control the mouse and other operations in the system

System featuring a 96-segment LCD display, an integrated pressure sensor, and a three-axis accelerometer for motion sensitive control. The integrated wireless interface allows the MSP430 microcontroller to act as a central hub for nearby wireless sensors such as pedometers and heart-rate monitors.

The MSP430 microcontroller offer temperature and battery voltage measurement and is complete with a USB-based MSP430F5509 + CC1101 (part of the new MSP430 microcontroller kit with white PCBs) or CC1111 (part of the initial eZ430- Chronos kit with black PCBs) wireless interface to a PC. The MSP430 microcontroller wrist module may be disassembled to be programmed with custom applications and includes an eZ430 USB programming interface.

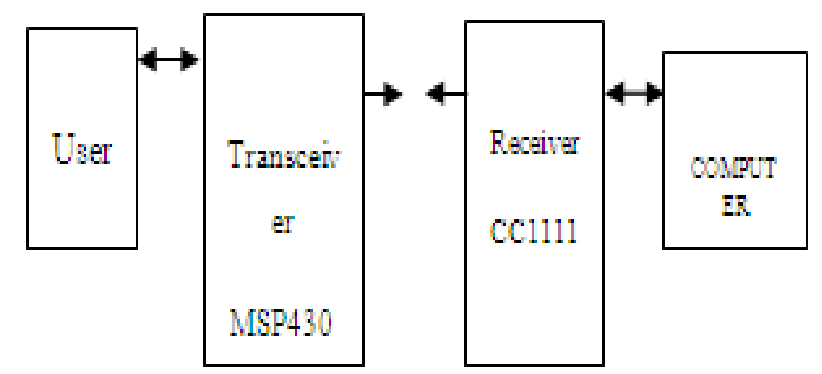

Figure 4: Proposed model

\section{1) Description}

The CC1110Fx, CC1111Fx is a true low-power sub- $1 \mathrm{GHz}$ system-on-chip (SoC) designed for low power wireless applications. The $\mathrm{CC} 1110 \mathrm{Fx}, \mathrm{CC} 1111 \mathrm{Fx}$ combines the excellent performance of the state-of-theart $\mathrm{RF}$ transceiver $\mathrm{CC} 1101$ with an

\section{2) PC Mouse Control}

Click Mouse on (M) to control the PC mouse pointer with the MSP430 microcontroller module. Hold the Chronos module with its display facing up. The mouse pointer moves vertically ( $\mathrm{x}$-axis in Control Center) 
when tilting the Chronos module forward/backward and moves vertically (y-axis in Control Center) when tilting the Chronos module left/right. Mouse clicks are possible as well:

- Left single click: * button

- Left double click: \# button

- Right click: UP button

Mouse control can be calibrated to set a point of zero acceleration (that is, no pointer movement) by selecting Calibration (C). It may be disabled by clicking Mouse Off (M) or by typing M on the PC keyboard. NOTE: Holding buttons (for example, for drag and drop) is not supported. Turn the demo off by pushing the DOWN button on the MSP430 microcontroller module and clicking Stop Access Point in the PC application.

\section{3) PowerPoint Control}

The Control Center allows the user to map button pushes on the wrist module into keystrokes on the PC. The default setting is PowerPoint control, which allows switching slides forward/backward and to start the slide show.

1. Select the SimpliciTI Acc/PPT tab.

2. Click Start Access Point to start linking. The control center status line displays "Access point started".

3. Select PPt mode in the bottom LCD line of the MSP430 microcontroller module and activate the RF link by pressing the DOWN button. The wrist module connects to the PC, this may take a moment.

4. Once connected, the Control Center status bar show when a button is pushed.

5. Open a PowerPoint presentation. Press \# to go to presentation mode (slide show - F5), UP to switch to next slide (right arrow key), and * (left arrow key) to switch to previous slide.

\section{4) Functional Description of the MSP430 microcontroller Wrist Module}

The core technology behind the MSP430 microcontroller wrist module is the CC430F6137 microcontroller with its integrated $<1-\mathrm{GHz}$ radio. The CC430 also controls the LCD and its temperature sensor is used for temperature measurement. The only other ICs on the Chronos module PCB are pressure and acceleration sensors and the LCD backlight driver.

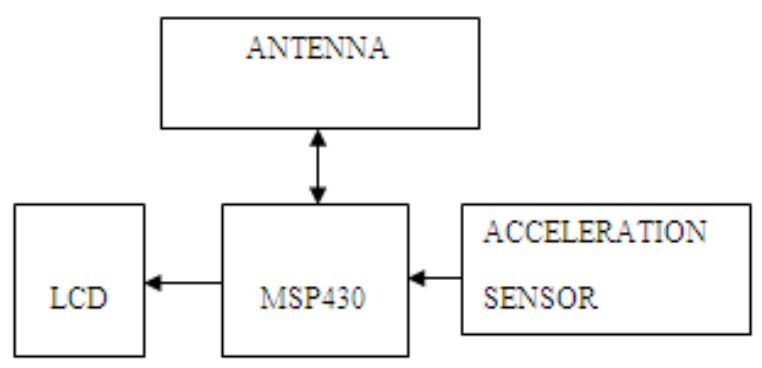

Figure 5 Wrist Module Block Diagram

\section{Results}

In this project we implemented the mouse control in two ways .First Method is based on RGB color detection which are at the tip of the user's fingers. Marking the user's fingers with red, green, and blue tape helps the webcam recognize gestures. The movements and arrangements of these Markers are interpreted into gestures that act as interaction instructions for the projected application interface.

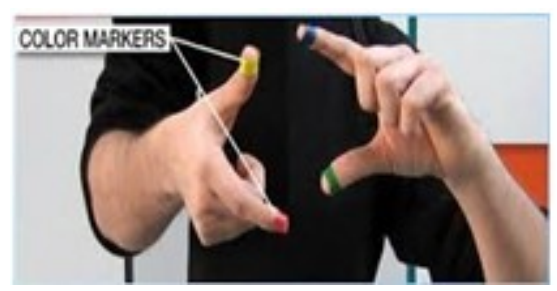

Figure 6 Color Markers 


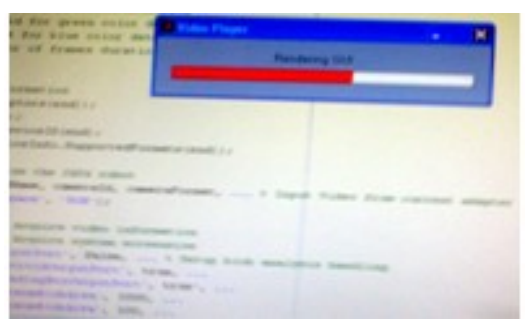

Figure7 Building GUI

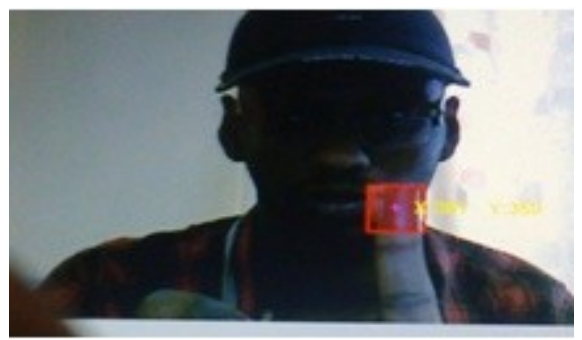

Figure 8: Tracing Red

Red Squares indicates the detected region of Blue color which indicates single blue for Right click and Two Blue indicates Left click of mouse is done .It also indicates the region of interest.

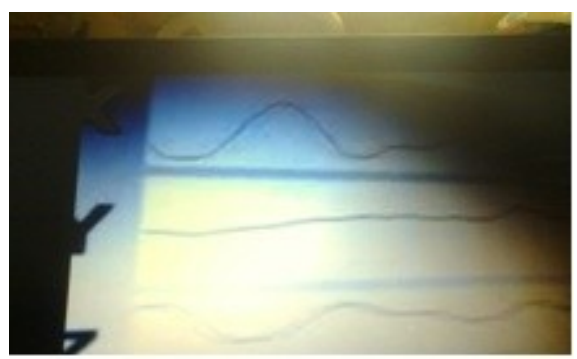

Figure 9: 3D- Mouse axis

In Cognitive Mouse experiment we used MSP430 microcontroller as a Transmitter and CC111 USB $\mathrm{RF}$ as Receiver. The Receiver RF USB is attached to computer.

\section{Applications:}

- Fixed Function used to communicate between User and PC

- Accuracy, Time to Act

- Multi-Functional with Cognitive Mouse.

\section{Conclusion:}

In this study, an object tracking based virtual mouse application has been developed and implemented using a webcam. The system has been implemented in MATLAB environment using MATLAB Image Processing Toolbox. As an object a blue color sticker is used to make the detection easy and fast. Object detection and motion tracking worked very well. Using the pointer moving the cursor and the simulating the mouse click events also worked well. However, system has some disadvantages such as being invariant to illumination up to some scale, and movement of the cursor is very sensitive to motion. Because of this reason, to control the cursor, pointer cannot be used on the air efficiently.

Cognitive Mouse is implemented with the help of MSP430 microcontroller which will perform MultiFunctions like Mouse Pointer Controlling, PPT Controlling. It overcomes the Disadvantages with virtual mouse .It had better Accuracy. Using the pointer moving the cursor and the simulating the mouse click events also worked well.

\section{Bibliography}

[1]. Bare Finger Touch Detection by the Button's Distortion in Projector-Camera System by Jun Hu, Guolin Li, Xiang Xie, Zhong Lv, Zhihua Wang, Senior, IEEE -2013

[2]. Y. Sato,Y. Kobayashi,H. Koike "Fast tracking of hands and fingertips in infrared images foraugmented desk interface",2000

[3]. Z. Zhang, Y. Wu, Y.Shan, S. Shafer "Visual panel: Virtual mouse keyboard detection" July, 2003

[4]. J. David and Z. David, "A Survey of Glove-based Input," IEEE Computer Graohics \& Applications, vol. 4, January 1994, pp.30-39.

[5]. Fifth Dimension Technologies, 5DT Data Glove for the Fifth Dimension User's Manual [Z], February 2000 


\section{Implementation of Cognitive and Virtual Mouse Pointer Control}

[6]. Robert Y. Wang and Jovan Popovic, "Real-Time Hand-Tracking with a color Glove," ACM Transactions on Graphics, vol. 28, no. 3, 2009.

[7]. Y. Wu and T.S. Huang, "Hand modeling, analysis and recognition for vision-based human computer interaction," IEEE Signal Processing Magazine, vol. 18, May 2001, pp:51-60.

[8]. M.Lee, R. Green and M.Billinghurst, "3D Natural Hand Interaction for AR Applications," Image and Vision Computer New Zealand, 23rd International Conference, 2008

[9]. M. Gorman, M. Betke, M. Saltzman, and A. Lahav, "Music Maker -A Camera-based Music Making Tool for Physical Rehabilitation," Boston University Computer Science Technical Report N0.2005-032,2005

[10]. www.mathworks.com 